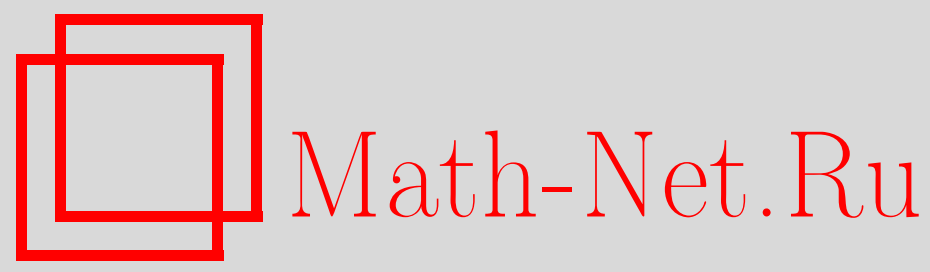

C. В. Асташкин, О пространстве мультипликаторов, порожденном системой Радемахера, Матем. заметки, 2004, том 75, выпуск 2, 173-181

DOI: https://doi.org/10.4213/mzm26

Использование Общероссийского математического портала Math-Net.Ru подразумевает, что вы прочитали и согласны с пользовательским соглашением http://www . mathnet.ru/rus/agreement

Параметры загрузки:

IP : 18.207 .199 .55

26 апреля 2023 г., 15:10:56 


\section{О ПРОСТРАНСТВЕ МУЛЬТИПЛИКАТОРОВ, ПОРОЖДЕННОМ СИСТЕМОЙ РАДЕМАХЕРА}

\section{С. В. Асташкин}

В работе рассматривается пространство мультипликаторов симметричного пространства относительно системы Радемахера. Найдены достаточные условия, при которых оно совпадает с пространством $L_{\infty}$ (с эквивалентностью норм). Условия формулируются в терминах теории интерполяции операторов и являются существенно более слабыми, нежели условия, полученные недавно при решении этой задачи другими авторами.

Библиография: 11 названий.

1. Введение. Пусть $X$ - симметричное пространство функций, измеримых на $[0,1]$. Следуя работе [1], через $\Lambda(R, X)$ обозначим множество всех измеримых $f:[0,1] \rightarrow \mathbb{R}$ таких, что $f g \in X$ для всех функций вида $g(t)=\sum_{k=1}^{\infty} c_{k} r_{k}(t)$, где $r_{k}(t), k=1,2, \ldots,-$ функции Радемахера, т.е.

$$
r_{k}(t)=\operatorname{sign} \sin \left(2^{k} \pi t\right) \quad(t \in[0,1]) .
$$

Тогда $\Lambda(R, X)$ - банахова функциональная решетка относительно нормы

$$
\|f\|_{\Lambda}=\sup \left\{\|f g\|_{X}: g=\sum_{k=1}^{\infty} c_{k} r_{k} \in X,\|g\|_{X} \leqslant 1\right\} .
$$

Иначе говоря, это пространство мультипликаторов в $X$, ограниченно действующих из замкнутой линейной оболочки $\left[r_{k}\right]_{X}$, натянутой на систему Радемахера, во все пространство.

В [1] было показано, что для широкого класса симметричных пространств $X$ таких, что $\left[r_{k}\right]_{X}$ изоморфно $l_{2}$ (необходимые и достаточные условия, при которых это вьполнено, были получены в работе [2]), пространство $\Lambda(R, X)$ не симметрично. Точнее, из принадлежности функции $f(t)$ к $\Lambda(R, X)$, вообще говоря, не следует, что к $\Lambda(R, X)$ принадлежит и невозрастающая перестановка ее модуля $f^{*}(t)$. В то же время оказалось, что при некоторых условиях $\Lambda(R, X)=L_{\infty}$ с эквивалентностью норм [3], [4]. Для формулировки этих условий нам понадобится следуюший квазилинейньй оператор, введенный ранее также при изучении системы Радемахера в работе [5].

На множестве всех измеримых функций $f:[0,1] \rightarrow \mathbb{R}$ определим оператор

$$
Q f(t)=\left\{\frac{1}{\theta(t)} \int_{0}^{t}\left(f^{*}(s)\right)^{2} d \theta(s)\right\}^{1 / 2}, \quad \text { где } \theta(t)=\ln ^{-1} \frac{e}{t} .
$$

Сформулируем теперь основное утверждение из работ [3], [4]. 
Теорема (Г. П. Курбера, В. А. Родин). Если оператор $Q$ ограниченно действует в симметричном пространстве $X$, то

$$
\Lambda(R, X)=L_{\infty}
$$

\section{с әквивалентностью норм.}

Здесь равенство (2) будет доказано для пространств, интерполяционьх относительно банаховой пары $\left(L_{\infty}, L_{N}\right)$, где $L_{N}$ - пространство Орлича, построенное по функции $N(u)=e^{u^{2}}-1$ (теорема 1). В доказательстве мы используем описание подпространств вида $\left[r_{k}\right]_{X}$ для таких пространств, полученное ранее в работах автора [6], [7]. Условие интерполяционности относительно банаховой пары $\left(L_{\infty}, L_{N}\right)$ менее ограничительно, нежели ограниченность оператора $Q$ (см. теорему 2 и замечание 1 ). Поэтому теорема 1 усиливает приведенную выше теорему Курберы и Родина.

2. Определения и обозначения. Подробное изложение теории симметричных функциональных пространств и теории интерполяции операторов можно найти, например, в монографиях [8]-[10].

Если функция $x=x(t)$ измерима на $[0,1]$, то по определению

$$
n_{|x|}(\tau)=\mu\{t \in[0,1]:|x(t)|>\tau\} \quad \text { для } \tau>0
$$

( $\mu$-мера Лебега). Функции $x(t)$ и $y(t)$ назьваются равноизмеримымми, если $n_{|x|}(\tau)=$ $n_{|y|}(\tau), \tau>0$. В частности, любая функция $x(t)$ равноизмерима со своей невозрастающей перестановкой

$$
x^{*}(t)=\inf \left\{\tau \geq 0: n_{|x|}(\tau)<t\right\} .
$$

Банахово пространство $X$ измеримых функций, определенных на $[0,1]$, назьвается симметричным пространством (СП), если выполнены следуюшие условия:

а) из того, что $y=y(t) \in X$ и $|x(t)| \leqslant|y(t)|$, следует, что $x=x(t) \in X$ и $\|x\| \leqslant\|y\|$;

б) если $y=y(t) \in X$ и функции $x=x(t)$ и $y=y(t)$ равноизмеримы, то $x \in X$ и $\|x\|=\|y\|$.

Фундаментальная функиия СП $X$ определяется равенством $\varphi_{X}(t)=\left\|\chi_{(0, t)}\right\|_{X}$, где, как обычно, $\chi_{G}(s)=1$, если $s \in G$, и $\chi_{G}(s)=0$, если $s \notin G$. Напомним, что для любого СП функция $\varphi_{X}(t)$ квазивогнута на $(0,1][8$, с. 137$]$, т.е. неотрицательна, возрастает, а функция $\varphi_{X}(t) / t$ убьвает на этом отрезке. Как известно $[8$, с. 70$]$, каждая квазивогнутая функция $f(t)$ эквивалентна своей наименьшей вогнутой мажоранте $\tilde{f}(t)$, точнее,

$$
f(t) \leqslant \tilde{f}(t) \leqslant 2 f(t), \quad t \in[0,1] .
$$

В каждом СП $X$ непрерьвен оператор растяжения $\sigma_{\tau} x(t)=x(t / \tau) \chi_{[0,1]}(t / \tau), \tau>0$ [8, с. 131-133]. Более того, для всех $\tau>0$

$$
\left\|\sigma_{\tau}\right\|_{X \rightarrow X} \leqslant \max (1, \tau) .
$$

Важным примером СП является пространство Орлича. Пусть $M(t)$ - возрастающая выпуклая функция на $[0, \infty), M(0)=0$. Пространство Орлича $L_{M}$ состоит из всех измеримых на $[0,1]$ функций $x=x(t)$ таких, что норма

$$
\|x\|_{L_{M}}=\inf \left\{u>0: \int_{0}^{1} M\left(\frac{|x(t)|}{u}\right) d t \leq 1\right\}
$$


конечна. В частном случае $M(t)=t^{p}, 1 \leqslant p<\infty$, получаем обычные $L_{p}$-пространства. Непосредственное вычисление показывает, что фундаментальная функция $\varphi_{L_{M}}(t)=$ $1 / M^{-1}(1 / t)$, где $M^{-1}(u)$ - функция, обратная к $M(u)$.

Пусть $\psi(t)$ - квазивогнутая функция на $[0,1]$. Пространство Мариинкевича $M(\psi)$ состоит из всех измеримых на $[0,1]$ функций $x=x(s)$, для которых

$$
\|x\|_{M(\psi)}=\sup _{0<t \leq 1} \frac{\int_{0}^{t} x^{*}(s) d s}{\psi(t)}<\infty .
$$

Заметим, что это пространство является сопряженным к пространству Лоренца $\Lambda(\tilde{\psi})[8$, c. 152-154],

$$
\|x\|_{\Lambda(\tilde{\psi})}=\int_{0}^{1} x^{*}(s) d \tilde{\psi}(s) .
$$

$\Phi$ ундаментальная функция пространства $M(\psi)$ равна $t / \psi(t)$.

Пусть $\left(X_{0}, X_{1}\right)$ - банахова пара, т.е. пара банаховых пространств, линейно и непрерьвно вложенных в некоторое отделимое линейное топологическое пространство, $x \in X_{0}+X_{1}, t>0$. Определим $\mathscr{K}$-функиионал Петре

$$
\mathscr{K}\left(t, x ; X_{0}, X_{1}\right)=\inf \left\{\left\|x_{0}\right\|_{X_{0}}+t\left\|x_{1}\right\|_{X_{1}}: x=x_{0}+x_{1}, \quad x_{0} \in X_{0}, \quad x_{1} \in X_{1}\right\} .
$$

Этот функционал играет важную роль в теории интерполяции операторов и в некоторых других разделах анализа. При фиксированном $x \in X_{0}+X_{1} \mathscr{K}$-функционал-возрастающая вогнутая функция относительно переменной $t[9$, гл. 3 , лемма 3.1.1]. Если же зафиксировать $t>0$, то $\mathscr{K}\left(t, x ; X_{0}, X_{1}\right)$ является нормой на сумме пространств $X_{0}+t X_{1}$.

Банахово пространство $X$ назьвается интерполяиионным относительно банаховой пары $\left(X_{0}, X_{1}\right)$, если $X_{0} \cap X_{1} \subset X \subset X_{0}+X_{1}$ и из ограниченности линейного оператора в пространствах $X_{0}$ и $X_{1}$ следует его ограниченность в $X$.

Одним из важнейших способов получения интерполяционных пространств является так назьваемьй $\mathscr{K}$-метод, основанный на использовании $\mathscr{K}$-функционала Петре.

Пусть $F$ - банахова решетка двусторонних числовых последовательностей

$$
a=\left(a_{k}\right)_{k=-\infty}^{\infty}
$$

$F \supset l_{\infty} \cap l_{\infty}\left(2^{-k}\right)$ (если $1 \leqslant p \leqslant \infty$ и $w_{k}>0, k=0, \pm 1, \ldots$, то “весовое" пространство $l_{p}\left(w_{k}\right)$ состоит из всех $a=\left(a_{k}\right)_{k=-\infty}^{\infty}$ таких, что $\left(a_{k} w_{k}\right)_{k} \in l_{p}$ и $\|a\|_{l_{p}\left(w_{k}\right)}=$ $\left.\left\|\left(a_{k} w_{k}\right)\right\|_{l_{p}}\right)$. Если $\left(X_{0}, X_{1}\right)$ - произвольная банахова пара, то пространство $\mathscr{K}$-метода $\left(X_{0}, X_{1}\right)_{F}^{\mathscr{K}}$ состоит из всех $x \in X_{0}+X_{1}$, для которых последовательность

$$
\left(\mathscr{K}\left(2^{k}, x ; X_{0}, X_{1}\right)\right)_{k} \in F,
$$

а норма элемента в нем задается равенством

$$
\|x\|=\left\|\left(\mathscr{K}\left(2^{k}, x ; X_{0}, X_{1}\right)\right)_{k}\right\|_{F} .
$$

Нетрудно показать, что пространство $\left(X_{0}, X_{1}\right)_{F}^{\mathscr{K}}$ интерполяционно относительно пары $\left(X_{0}, X_{1}\right)$.

В частности, для $0<\theta<1,1 \leqslant p \leqslant \infty$ получим классические пространства $\left(X_{0}, X_{1}\right)_{\theta, p}=\left(X_{0}, X_{1}\right)_{l_{p}\left(2^{-k \theta}\right)}^{\mathscr{K}}$. Свойства их подробно рассматриваются в монографии $[9]$.

В заключение скажем несколько слов об обозначениях. Выражение вида $F_{1} \asymp F_{2}$ в дальнейшем означает, что для некоторых $C_{1}>0$ и $C_{2}>0$ выполнены оценки $C_{1} F_{1} \leqslant$ $F_{2} \leqslant C_{2} F_{1}$, причем константы $C_{1}$ и $C_{2}$, как правило, не зависят от всех или части аргументов $F_{1}$ и $F_{2}$. 
3. Вспомогательные результаты. Получим сначала подходящее выражение для $\mathscr{K}$-функционала $\mathscr{K}\left(t, f ; L_{\infty}, L_{N}\right)$, где $L_{N}$ - пространство Орлича, построенное по функции $N(u)=e^{u^{2}}-1$.

Пусть $\psi(t)$ и $\varphi(t)$ - две возрастающие вогнутые неотрицательные функции на $[0,1]$. Ввиду $\left[8\right.$, с. 165-166] норма пересечения $\Lambda(\psi) \cap t^{-1} \Lambda(\varphi)$, равная по определению $\max \left(\|f\|_{\Lambda(\psi)}, t^{-1}\|f\|_{\Lambda(\varphi)}\right)$, эквивалентна норме пространства Лоренца $\Lambda\left(\max \left(\psi, t^{-1} \varphi\right)\right)$. Поэтому, переходя к сопряженным пространствам [8, с. 27] и учитьвая (3), получаем

$$
\mathscr{K}(t, f ; M(\psi), M(\varphi)) \asymp \sup _{0<u \leqslant 1} \frac{\int_{0}^{u} f^{*}(s) d s}{\max \left(\psi(u), t^{-1} \varphi(u)\right)}
$$

с константами, не зависящими от $t>0$ и $f \in M(\psi)+M(\varphi)$.

ЛЕмма 1. С константами, не зависящими от $t>0 u f \in L_{N}$, выполнено

$$
\mathscr{K}\left(t, f ; L_{\infty}, L_{N}\right) \asymp t \sup _{0<u \leqslant \min \left(1,2^{1-t^{2}}\right)}\left(f^{*}(u) \log _{2}^{-1 / 2} \frac{2}{u}\right) .
$$

ДокАЗАТЕЛЬСТво. Прежде всего заметим, что пространство $L_{N}$ одновременно является (с эквивалентностью норм) пространством Марцинкевича $M\left(\psi_{1}\right)$ для $\psi_{1}(u)=$ $u \log _{2}^{1 / 2}(2 / u)[11]$. Кроме того, очевидно, $L_{\infty}=M\left(\psi_{0}\right), \psi_{0}(u)=u$. Поэтому из соотношения (5) следует, что

$$
\mathscr{K}\left(t, f ; L_{\infty}, L_{N}\right) \asymp\|f\|_{M\left(\psi_{t}\right)}, \quad \text { где } \quad \psi_{t}(u)=u \max \left\{1, t^{-1} \log _{2}^{1 / 2} \frac{2}{u}\right\} .
$$

Пусть $\mathscr{M}_{\psi_{t}}(s)$ - функция растяжения функции $\psi_{t}$, т.е.

$$
\mathscr{M}_{\psi_{t}}(s)=\sup _{0<u \leqslant \min (1,1 / s)} \frac{\psi_{t}(s u)}{\psi_{t}(u)} .
$$

Представим

$$
\mathscr{M}_{\psi_{t}}\left(\frac{1}{2}\right)=\frac{1}{2} \sup _{0<u \leqslant 1} F_{t}(u), \quad \text { где } \quad F_{t}(u)=\frac{\max \left(1, t^{-1} \log _{2}^{1 / 2}(4 / u)\right)}{\max \left(1, t^{-1} \log _{2}^{1 / 2}(2 / u)\right)} .
$$

Так как $\log _{2}^{1 / 2}(4 / u) \leqslant \sqrt{2} \log _{2}^{1 / 2}(2 / u)$, то $F_{t}(u) \leqslant \sqrt{2}$. Поэтому для любого $t>0$ имеем $\mathscr{M}_{\psi_{t}}(1 / 2) \leqslant 1 / \sqrt{2}$ и, значит,

$$
\begin{aligned}
\|f\|_{M\left(\psi_{t}\right)} \asymp \sup _{0<u \leqslant 1}\left\{f^{*}(u) \min \left(1, t \log _{2}^{-1 / 2} \frac{2}{u}\right)\right\} \\
=t \sup _{0<u \leqslant \min \left(1,2^{1-t^{2}}\right)}\left(f^{*}(u) \log _{2}^{-1 / 2} \frac{2}{u}\right)
\end{aligned}
$$

с константами, не зависящими от $t>0$ и $f[8$, с. 156]. Следовательно, ввиду (6) лемма доказана. 
Далее нам понадобится следующее соотношение для $\mathscr{K}$-функционала $\mathscr{K}_{1,2}(t, a)=$ $\mathscr{K}\left(t, a ; l_{1}, l_{2}\right)$, построенного по банаховой паре $\left(l_{1}, l_{2}\right)$, с некоторой константой $c>0$, не зависящей от $a=\left(a_{k}\right)_{k=1}^{\infty} \in l_{2}$ и $t>0[11]$ :

$$
c\left(\sum_{i=1}^{\left[t^{2}\right]} a_{i}^{*}+t\left(\sum_{i=\left[t^{2}\right]+1}^{\infty}\left(a_{i}^{*}\right)^{2}\right)^{1 / 2}\right) \leqslant \mathscr{K}_{1,2}(t, a) \leqslant \sum_{i=1}^{\left[t^{2}\right]} a_{i}^{*}+t\left(\sum_{i=\left[t^{2}\right]+1}^{\infty}\left(a_{i}^{*}\right)^{2}\right)^{1 / 2}
$$

где $\left(a_{i}^{*}\right)_{i=1}^{\infty}-$ убьвающая перестановка последовательности $\left(\left|a_{k}\right|\right)_{k=1}^{\infty}, \mathrm{a}[z]-$ целая часть числа $z$.

Пусть $n \in \mathbb{N}$ и $b_{n}=(1 / n) \sum_{k=1}^{n} e_{k}$, где $e_{k}$ - стандартные орты в пространстве числовых последовательностей.

Лемма 2. С константами, не зависящими от $t>0$ и $n \in \mathbb{N}$,

$$
\mathscr{K}_{1,2}\left(t, b_{n}\right) \asymp \min \left(1, \frac{t}{\sqrt{n}}\right)
$$

ДокАЗАтЕльство. Пусть сначала $t \in \mathbb{N}$. Тогда, если $t^{2} \geqslant n$, то $(8)-$ непосредственное следствие соотношения (7). Пусть теперь $t^{2}<n$. Тогда ввиду (7)

$$
c\left(v^{2}+v\left(1-v^{2}\right)^{1 / 2}\right) \leqslant \mathscr{K}_{1,2}\left(t, b_{n}\right) \leqslant v^{2}+v\left(1-v^{2}\right)^{1 / 2},
$$

где $v=t / \sqrt{n}<1$. В случае $v \leqslant 1 / \sqrt{2}$ отсюда следует

$$
\frac{c}{\sqrt{2}} v \leqslant \mathscr{K}_{1,2}\left(t, b_{n}\right) \leqslant v^{2}+v \leqslant\left(1+\frac{1}{\sqrt{2}}\right) v
$$

Если же $1 / \sqrt{2}<v<1$, то опять

$$
\frac{c}{\sqrt{2}} v \leqslant c v^{2} \leqslant \mathscr{K}_{1,2}\left(t, b_{n}\right) \leqslant 2 v .
$$

Тем самым (8) доказано для натуральных, а значит, и для всех $t \geqslant 1$ ввиду квазивогнутости $\mathscr{K}$-функционала по $t$. Осталось заметить, что при $0<t \leqslant 1$ по определению $\mathscr{K}$-функционала

$$
\mathscr{K}_{1,2}\left(t, b_{n}\right)=t\left\|b_{n}\right\|_{l_{2}}=v
$$

Лемма доказана.

\section{4. Основные результаты.}

Теорема 1. Если $X$ - пространство, интерполяционное относительно банаховой пары $\left(L_{\infty}, L_{N}\right)$, то $\Lambda(R, X)=L_{\infty}($ с әквивалентностью норм $)$. 
ДокАЗАТЕЛЬство. Пусть $h_{n}(t)=\chi_{\left[0,2^{-n}\right]}(t), n \in \mathbb{N}$. По лемме 1 с константами, не зависящими от $n \in \mathbb{N}$ и $k=0, \pm 1, \pm 2, \ldots$,

$$
\mathscr{K}\left(2^{k}, h_{n} ; L_{\infty}, L_{N}\right) \asymp 2^{k} \min \left(\frac{1}{\sqrt{n}}, \frac{1}{2^{k}}\right) .
$$

Следовательно, если, как и ранее, $b_{n}=(1 / n) \sum_{i=1}^{n} e_{i}$, то ввиду леммы 2

$$
\mathscr{K}\left(2^{k}, h_{n} ; L_{\infty}, L_{N}\right) \asymp \mathscr{K}_{1,2}\left(2^{k}, b_{n}\right)
$$

с константами, не зависящими от $n \in \mathbb{N}$ и $k=0, \pm 1, \pm 2, \ldots$

По условию пространство $X$ - интерполяционное пространство относительно банаховой пары $\left(L_{\infty}, L_{N}\right)$ и, значит,

$$
X=\left(L_{\infty}, L_{N}\right)_{F}^{\mathscr{K}}
$$

для некоторой банаховой решетки $F$ двусторонних числовых последовательностей $[6],[7]$. Поэтому если $\varphi_{X}(t)-$ фундаментальная функция $X$, то из (9) следует

$$
\varphi_{X}\left(2^{-n}\right) \asymp\left\|\left(\mathscr{K}\left(2^{k}, h_{n} ; L_{\infty}, L_{N}\right)\right)_{k}\right\|_{F} \asymp\left\|\left(\mathscr{K}_{1,2}\left(2^{k}, b_{n}\right)\right)_{k}\right\|_{F} .
$$

С другой стороны [6], [7],

$$
\left\|\frac{1}{n} \sum_{i=1}^{n} r_{i}\right\|_{X} \asymp\left\|\left(\mathscr{K}_{1,2}\left(2^{k}, b_{n}\right)\right)_{k}\right\|_{F} .
$$

Таким образом,

$$
\left\|\sum_{i=1}^{n} r_{i}\right\|_{X} \asymp n \varphi_{X}\left(2^{-n}\right) .
$$

Пусть теперь $G \subset[0,1]$ - произвольное измеримое множество, $\mu(G)>0$. Так как почти все точки измеримого множества - точки плотности относительно меры Лебега, можно найти двоичньй интервал $I$ такой, что

$$
\mu(G \cap I) \geqslant \frac{1}{2} \mu(I) .
$$

Предположим, что ранг $I$ равен $n$, т.е. $I=\left[(i-1) 2^{-n}, i 2^{-n}\right)$ для некоторого $i=$ $1,2, \ldots, 2^{n}$. Выберем $\varepsilon_{i}= \pm 1, i=1,2, \ldots, n$, так, что при всех $t \in I$ вьполнено $\sum_{i=1}^{n} \varepsilon_{i} r_{i}(t)=n$. Тогда, если $K=\left\|\sum_{i=1}^{n} \varepsilon_{i} r_{i}\right\|_{X}$, то из определения $\Lambda(R, X)$, симметричности $X$, неравенства $\left\|\sigma_{\tau}\right\|^{-1} \leqslant\left\|\sigma_{\tau^{-1}}\right\|$, а также из соотношений (4) и (11) получаем

$$
\begin{aligned}
\left\|\chi_{G}\right\|_{\Lambda} & \geqslant\left\|\chi_{G \cap I}\right\|_{\Lambda} \geqslant \frac{1}{K}\left\|\chi_{G \cap I} \sum_{i=1}^{n} \varepsilon_{i} r_{i}\right\|_{X} \\
& \geqslant \frac{1}{K}\left\|\sigma_{2}\right\|_{X \rightarrow X}^{-1}\left\|\chi_{I} \sum_{i=1}^{n} \varepsilon_{i} r_{i}\right\|_{X} \geqslant \frac{n}{2 K} \varphi_{X}\left(2^{-n}\right) .
\end{aligned}
$$


В силу определения функций Радемахера и симметричности пространства $X$

$$
K=\left\|\sum_{i=1}^{n} r_{i}\right\|_{X}
$$

Поэтому из последнего неравенства и (10) следует

$$
\left\|\chi_{G}\right\|_{\Lambda(R, X)} \geqslant c_{0}
$$

где константа $c_{0}>0$ не зависит от множества $G$.

Так как $L_{\infty} \subset \Lambda(R, X)$, то для доказательства теоремы достаточно показать, что пространство мультипликаторов $\Lambda(R, X)$ не содержит ни одной неограниченной функции. Предположим, что $f \in \Lambda(R, X)$ и неограничена. Тогда для каждого $n \in \mathbb{N}$ мера множества

$$
G_{n}=\{t \in[0,1]:|f(t)| \geqslant n\}
$$

положительна. Поэтому, так как $\Lambda(R, X)$ - банахова решетка, из (12) следует

$$
\|f\|_{\Lambda} \geqslant n\left\|\chi_{G_{n}}\right\|_{\Lambda} \geqslant n c_{0} \quad \text { для любого } n \in \mathbb{N} \text {. }
$$

Последнее противоречит тому, что $f \in \Lambda(R, X)$. Теорема доказана.

При изучении вопросов, связанньх с системой Радемахера в СП (см. [3]-[5]) весьма полезным оказался квазилинейньй оператор $Q$, определенньй соотношением (1).

В [4] показано, что если оператор $Q$ ограниченно действует в СП $X$, то $L_{\infty} \subset X \subset L_{N}$. Здесь мы докажем более сильное утверждение.

Теорема 2. Если оператор $Q$ ограниченно действует в СП $X$, то пространство $X$ интерполяиионно относительно банаховой пары $\left(L_{\infty}, L_{N}\right)$.

ДокАЗАТЕЛЬСТво. Введем пространство Лоренца $\Lambda_{2}(\theta)$ с нормой

$$
\|f\|_{\Lambda_{2}(\theta)}=\left\{\int_{0}^{1}\left(f^{*}(s)\right)^{2} d \theta(s)\right\}^{1 / 2}, \quad \theta(t)=\ln ^{-1} \frac{e}{t},
$$

а также класс $\Sigma$ всех линейных операторов, ограниченных из $L_{\infty}$ в $L_{\infty}$ и из $\Lambda_{2}(\theta)$ в $L_{N}$. Покажем, что линейньй оператор $T \in \Sigma$ тогда и только тогда, когда существует константа $C>0$ такая, что при всех $f \in \Lambda_{2}(\theta)$ и $0<t \leqslant 1$

$$
(T f)^{*}(t) \leqslant C \mathscr{K}\left(\log _{2}^{1 / 2} \frac{2}{t}, f ; L_{\infty}, \Lambda_{2}(\theta)\right) .
$$

Как уже говорилось в доказательстве леммы 1 , пространство $L_{N}$ совпадает с пространством Марцинкевича $M\left(\psi_{1}\right), \psi_{1}(u)=u \log _{2}^{1 / 2}(2 / u)$. Более того (см. [8, с. 156] или лемму 1),

$$
\|f\|_{L_{N}} \asymp \sup _{0<s \leqslant 1}\left(f^{*}(s) \log _{2}^{-1 / 2} \frac{2}{s}\right) .
$$

Предположим, что $T \in \Sigma$. Представим $f \in \Lambda_{2}(\theta)$ в виде

$$
f=f_{0}+f_{1}, \quad \text { где } f_{0} \in L_{\infty}, \quad f_{1} \in \Lambda_{2}(\theta) .
$$


Учитьвая (4) и (14), для каждого $t>0$ получаем

$$
\begin{aligned}
(T f)^{*}(t) & \leqslant\left(\left|T f_{0}\right|+\left|T f_{1}\right|\right)^{*}(t) \leqslant\left(T f_{0}\right)^{*}\left(\frac{t}{2}\right)+\left(T f_{1}\right)^{*}\left(\frac{t}{2}\right) \\
& \leqslant C_{1} \max \left(\|T\|_{L_{\infty} \rightarrow L_{\infty}},\|T\|_{\Lambda_{2}(\theta) \rightarrow L_{N}}\right)\left(\left\|f_{0}\right\|_{L_{\infty}}+\left(\log _{2}^{1 / 2} \frac{2}{t}\right)\left\|f_{1}\right\|_{\Lambda_{2}(\theta)}\right)
\end{aligned}
$$

После перехода к точной нижней грани по всем представлениям (15) приходим к (13) с подходящей константой. Обратное утверж дение следует сразу из того, что

$$
\begin{aligned}
& \mathscr{K}\left(\log _{2}^{1 / 2} \frac{2}{t}, f ; L_{\infty}, \Lambda_{2}(\theta)\right) \leqslant\|f\|_{L_{\infty}}, \quad f \in L_{\infty}, \\
& \mathscr{K}\left(\log _{2}^{1 / 2} \frac{2}{t}, f ; L_{\infty}, \Lambda_{2}(\theta)\right) \leqslant\left(\log _{2}^{1 / 2} \frac{2}{t}\right)\|f\|_{L_{N}}, \quad f \in L_{N} .
\end{aligned}
$$

Стандартные рассуждения (см., например, [9, гл. 5, теорема 5.2.1]) показьвают, что

$$
\mathscr{K}\left(t, f ; L_{\infty}, \Lambda_{2}(\theta)\right) \asymp t\left\{\int_{0}^{2^{1-t^{2}}}\left(f^{*}(s)\right)^{2} d \theta(s)\right\}^{1 / 2}
$$

Поэтому ввиду (1) неравенство (13) эквивалентно следующему неравенству:

$$
(T f)^{*}(t) \leqslant C Q f(t)
$$

Заметим, что фундаментальная функция $\varphi_{\Lambda_{2}(\theta)}(t)=\theta^{1 / 2}(t) \asymp t / \psi_{1}(t)$. Следовательно [8, с. 160-162], вьполнены непрерывные вложения

$$
\Lambda\left(\frac{t}{\psi_{1}(t)}\right) \subset \Lambda_{2}(\theta) \subset M\left(\psi_{1}\right)=L_{N}
$$

Предположим теперь, что оператор $Q$ ограничен в СП $X$. Если $T$ - линейный оператор, ограниченный в $L_{\infty}$ и $L_{N}$, то ввиду вложений (17) $T \in \Sigma$, и значит, из неравенства (16) следует ограниченность $T$ в $X$. Таким образом, пространство $X$ интерполяционно относительно пары $\left(L_{\infty}, L_{N}\right)$.

ЗАмЕчаниЕ 1. Утверждение, обратное к только что доказанному, неверно. Например, пространство $X=L_{N}$, разумеется, интерполяционно относительно банаховой пары $\left(L_{\infty}, L_{N}\right)$, но, как нетрудно проверить [4], оператор $Q$ не ограничен в нем.

ЗАмЕчаниЕ 2. Теорема 2 показьвает, что теорема 1 , доказанная в этой работе, является усилением теоремы Курберы и Родина [3], [4], сформулированной во введении. 


\section{СПИСОК ЦИТИРОВАННОЙ ЛИТЕРАТУРЫ}

[1] Curbera G. P. A note on function spaces generated by Rademacher series // Proc. Edinburgh Math. Soc. 1997. V. 40. P. 119-126.

[2] Rodin V. A., Semenov E. M. Rademacher series in symmetric spaces // Analysis Math. 1975. V. 1. № 3. P. 207-222.

[3] Курбера Г. П., Родин В. А. О мульипликаторах на множестве рядов Радемахера // Тезисы 5-ой Казанской межд. школы-конференции. Казань, 2001. С. 148-149.

[4] Curbera G. P., Rodin V. A. Multiplication operators on the space of Rademacher series in rearrangement invariant spaces // Proc. Edinburgh Math. Soc. (to appear).

[5] Асташкин С. В., Семенов Е. М. Коэффициенты Фурье-Радемахера // Докл. РАН. 2000. T. 175. № 1. C. 7-9.

[6] Асташкин С. В. О рядах по системе Радемахера, "близких" к $L_{\infty} / /$ Функцион. анализ и его прилож. 1998. Т. 32. №3. С. 62-65.

[7] Асташкин С. В. Об интерполяции подпространств симметричных пространств, порожденных системой Радемахера // Изв. РАЕН. Сер. МММИУ. 1997. Т. 1. №1. С. 18-35.

[8] Крейн С. Г., Петунин Ю. И., Семенов Е. М. Интерполяция линейных операторов. M.: Наука, 1978.

[9] Берг Ц̆. , Лефстрем Ц̆. Интерполяционные пространства. Введение. М.: Мир, 1980.

[10] Lorentz G. G. Relations between function spaces // Proc. Amer. Math. Soc. 1961. V. 12. P. 127-132.

[11] Holmstedt T. Interpolation of quasi-normed spaces // Math. Scand. 1970. V. 26. P. 177-199. 